\title{
Two Liquid-Liquid Phase Transitions in Confined Water Nanofilms
}

Saeed Pourasad, ${ }^{1}$ Amir Hajibabaei, ${ }^{1}$ Chang Woo Myung, ${ }^{1}$ and Kwang S. Kim ${ }^{1 *}$

${ }^{1}$ Center for Superfunctional Materials, Department of Chemistry and Department of Physics, Ulsan National Institute of Science and Technology (UNIST), 50 UNIST-gil, Ulsan 44919, Republic of Korea.

*e-mail: kimks@unist.ac.kr

\section{Contents}

I. Methods
A. Simulation model
B. Method of simulation

\section{Mixing-driven Landau Phase Transitions due to High Order Interactions}
A. Free energy change $(\Delta G)$ due to entropy and enthalpy changes upon mixing
B. Symmetric $\Delta G$
C. Phase Transition in symmetric $\Delta G$
D. Asymmetric $\Delta G$
E. $\Delta G$ between different states with different chemical potentials
F. Mixing-driven enthalpy change due to high order Interactions

\section{Tables}

Table S1. Temperature dependent free energy change profiles for symmetric $\Delta G$

Table S2. Temperature dependent free energy change profiles for asymmetric $\Delta G$

\section{Figures}

Figure. S1. Time evolution of mean square displacement for two systems

Figure. S2. Autocorrelation functions for $Q_{6}$ and density

Figure. S3. Structural analysis of the states

Figure. S4. Comparison of ice and intermediate structures

Figure. S5. Bond orientational order parameter $Q_{6}$ with $N V T$ simulations

Figure. S6. Side views of the confined water at two different densities for different $\mathrm{z}$ distances.

V. References 


\section{Methods}

\section{A. Simulation model}

We performed simulations using the TIP4P/2005 model $^{1}$ for water. TIP4P/2005 is a re-parametrized version of the 'classical' TIP4P model ${ }^{2}$ and it is widely employed. In particular, it was shown to be the best rigid and non-polarizable potential for the reproduction of many features of experimental water ${ }^{3}$.

\section{B. Method of simulation}

The simulations were performed using LAMMPS package ${ }^{4}$. For water with $\rho \cong 1.065\left(\mathrm{gr} / \mathrm{cm}^{3}\right)$ and $\rho \cong$ $0.965\left(\mathrm{gr} / \mathrm{cm}^{3}\right)$ densities, 1280 water molecules were placed between two Lennard-Jones hydrophobic fixed walls $\left(\varepsilon_{\text {wall }}=0.092 \mathrm{kcal} / \mathrm{mol} \& \sigma_{\text {wall }}=3.262 \AA\right)$. More than one thousand molecules have been used to answer the controversial issue about the LLPT in water. We performed the simulations for a wide range of $\mathrm{LJ}$ walls distances from $\mathrm{h}_{\mathrm{LJ} \text {-walls }}=14.0 \AA$ to $20.0 \AA$ and report the cases of $15 \AA$ and $16 \AA$ as exemplary ones. We have used $12.0 \AA$ cutoff for Lennard-Jones interactions and the ParticleParticle Particle-Mesh (PPPM) method to compute long-range corrections to electrostatic interactions ${ }^{5}$.

The periodic boundary conditions are applied in the lateral direction ( $\mathrm{x}$ and $\mathrm{y}$ ) in parallel with the two fixed walls. Molecular dynamics simulations were conducted in the $N V T$ ensemble and the isothermal-isobaric $\left(N P_{L} T\right)$ ensemble using a Nosé-Hoover thermostat and barostat. We have used the lateral pressure $P_{L}\left(=P_{x x}=P_{y y}=P_{x y}\right)$ in all of our simulations. To calculate the super-cooled TIP4P/2005 water the simulated times varied between 0.1-0.5 $\mu$ s. The time-step was set to $1.0 \mathrm{fs}$.

For calculating the real $\mathrm{z}$ distance of the confined water in our systems we have calculated the average $\mathrm{O}-\mathrm{O}$ atoms distance between the top and bottom layers of water along the $\mathrm{z}$ direction and then we have added the $\mathrm{O}-\mathrm{H}$ bond distance and the $\mathrm{H}$ van der walls radius to it.

In this work we have used the free energy perturbation method (LAMMPS package support this free energy calculation method). Free energy perturbation theory, as presented by Zwanzig in his 1954 paper $^{6,7,8}$, relates the free energy difference between an initial (reference) and a final (target) state of a system to an average of a function of their energy difference evaluated by sampling for the initial state:

$$
\Delta F=F_{1}-F_{0}=-k T l n<\exp \left[\left(-\left(E_{1}-E_{0}\right)\right) / k T\right]>_{0}
$$

In practice, one runs a normal simulation for state A, but each time a new configuration is accepted, the energy for state B is also computed. The difference between states A and B may be in the atom types involved, in which case the $\Delta \mathrm{F}$ obtained is for "mutating" one molecule onto another, or it may be a difference of geometry, in which case one obtains a free energy map along one or more reaction coordinates. In our work the perturbation is by adding one water molecule to our system gradually. Actually in practice, the total change is typically broken down into multiple sub-steps. The start and end states of the chemical change to be investigated are imagined to lie on a non-physical coordinate, commonly called $\lambda$, so that a change from $\lambda=0$ to $\lambda=1(\Delta \lambda=0.1)$ (with 5 ns equilibration for each step) corresponds to a change from initial to final state. At the end we get the water free energy change at each thermodynamic points. We performed FEP calculations with $N P_{L} T$ simulation by changing $\lambda$ from 0 to 1 as a coupling parameter while we performed $\sim 5 \mathrm{~ns}$ simulations to reach the equilibration at each state (all the initial structures of our FEP calculations are from $N V T$ simulations-equilibrated 
structures and before performing the FEP calculations they had been equilibrated at the given pressure and temperature for around $100 \mathrm{~ns}$ ).

\section{Mixing-driven Landau Phase Transitions due to High Order Interactions.}

\section{A. Free energy change $(\Delta G)$ due to entropy and enthalpy changes upon mixing}

The free energy change ( $\Delta G$ ), (i.e., mixing free energy), upon mixing either between two components or between two different states of one component where their fractions are $\mathrm{x}$ and 1-x, is the sum of mixing-driven entropic energy change $(-T \Delta S)$ and mixing-driven enthalpy change $\left(\Delta H=\Delta H^{i}+\Delta E^{\mu}\right)$ which is composed of the interaction energy change $\left(\Delta H^{i}\right)$ and the chemical potential $(\mu)$ energy change $\left(\Delta E^{\mu}\right)$ of the components moieties:

$\Delta G=-T \Delta S+\Delta H$

Let $x=\frac{1}{2}+\frac{\epsilon}{2},|\epsilon| \leq 1$.

The minimum free energy change $\left(\Delta G_{\min }\right)$ is obtained with the condition of $\frac{\partial \Delta G}{\partial x} \equiv \Delta G_{x}=0$, or $\frac{\partial \Delta G}{\partial \epsilon} \equiv \Delta G_{\epsilon}=\frac{1}{2} \Delta G_{x}=0$.

The mixing-driven entropic energy change is given by

$-T \Delta_{\mathrm{S}}=k T x \ln x+k T(1-x) \ln (1-x)=k T\left(-\ln 2+\frac{\epsilon^{2}}{2}+\frac{\epsilon^{4}}{12}+\frac{\epsilon^{6}}{30}+\ldots\right)$.

$\Rightarrow \frac{\partial(-T \Delta S)}{\partial \epsilon}=k T \epsilon\left(1+\frac{\epsilon^{2}}{3}+\frac{\epsilon^{4}}{5}+\ldots\right)$,

where $k$ is Boltzmann constant.

The chemical potentials of two components or two states (A and B) which are $\mu_{A}$ and $\mu_{B}$, can be different when two different components are interchangeable by for example, chemical reaction, electron transfer, excitation, or configurational change. Then, we need to consider the chemical potential energy change upon mixing:

$\Delta E^{\mu}=x \mu_{A}+(1-x) \mu_{B}=\bar{\mu}+\frac{\mu_{A B}}{2} \epsilon$,

where $\bar{\mu}=\frac{\mu_{A}+\mu_{B}}{2}$ and $\mu_{A B}=\mu_{A}-\mu_{B}$

Then, we have

$\Delta G=-T \Delta S+\Delta H \equiv-T \Delta S+\Delta H^{i}+\Delta E^{\mu} \equiv \Delta G^{i}+\Delta E^{\mu}$.

However, for convenience's sake, we use $\Delta G^{i}$ simply as $\Delta G$ (without including $\Delta E^{\mu}$ ) for the time being:

$\Delta G=\Delta G^{i}=-T \Delta S+\Delta H^{i}$,

while we will reconsider $\Delta G=\Delta G^{i}+\Delta E^{\mu}$ near the end of our discussion.

For either many body or high order interaction driven energy change $\Delta H^{i}$, we here consider up to 6body or $6^{\text {th }}$-order interactions between two components or between two states in this equation. The mixing-driven enthalpy change due to two body to many body or $2^{\text {nd }}$-high order interactions is discussed at the end of this section.

Then, the mixing-driven enthalpy change is

$\Delta H^{i}=\sum_{m, n} \omega_{m, n} x^{m}(1-x)^{n}, m+n \leq 6$

or

$\Delta H^{i}=v_{2} x(1-x)+v_{4} x^{2}(1-x)^{2}+v_{6} x^{3}(1-x)^{3}+\ldots$

$+x(1-x)\left[v_{21} x+v_{31} x^{2}+v_{41} x^{3}+v_{51} x^{4}+\ldots\right]$

$+x^{2}(1-x)^{2}\left[v_{32} x+v_{42} x^{2}+\ldots\right]$,

where

$v_{2}=\omega_{11}+\omega_{12}+\omega_{13}+\omega_{14}+\omega_{15}$

$v_{4}=\omega_{22}+\omega_{23}+\omega_{24}-2 \omega_{13}-3 \omega_{14}-4 \omega_{15}$ 
$v_{6}=\omega_{33}-2 \omega_{24}+2 \omega_{15}$

$v_{i j}=\omega_{i j}-\omega_{j i}$ (which is generally small).

Note that overall there is a trend $\left|v_{2}\right| \gg\left|v_{4}\right| \gg\left|v_{6}\right|$ in most cases, though $v_{2}$ or $v_{4}$ can be zero or near-zero in some occasions. It should be noted that $v_{2}$ is not a pure two-body interaction of $\omega_{11}$ but an effective two-body interaction which includes $\omega_{12}, \omega_{13}, \omega_{14}, \omega_{15}, \cdots$. The additional interaction terms which cannot be represented into effective two-body interaction term of $v_{2}$, are represented as effective multi-body interaction terms in $v_{4}, v_{6} \cdots$.

In the symmetric case when $\omega_{i j}=\omega_{j i}$ (though $\omega_{i j}$ and $\omega_{j i}$ are somewhat similar but not necessarily same), the mixing-driven enthalpy change is simplified as

$\Delta H^{i}=v_{2} x(1-x)+v_{4} x^{2}(1-x)^{2}+v_{6} x^{3}(1-x)^{3}+\ldots$

By combining the mixing-driven entropic energy change and the mixing-driven enthalpy change, we have the mixing-driven free energy change (without including $\Delta G^{\mu}$ ):

$\Delta G=\Delta G^{i}=-\mathrm{T} \Delta \mathrm{S}+\Delta H^{i}$

$=\left[k T x \ln x+k T(1-x) \ln (1-x)+v_{2} x(1-x)+v_{4} x^{2}(1-x)^{2}+v_{6} x^{3}(1-x)^{3}+\ldots\right]_{\mathrm{sym}}$

$+\left\{x(1-x)\left[v_{21} x+v_{31} x^{2}+v_{41} x^{3}+v_{51} x^{4}+\ldots\right]+x^{2}(1-x)^{2}\left[v_{32} x+v_{42} x^{2}+\ldots\right]\right\}_{\text {asym }}$

$=\left[c_{0}+\frac{c_{2}}{2} \epsilon^{2}+\frac{c_{4}}{4} \epsilon^{4}+{\frac{c_{6}}{6}}^{6} \epsilon^{6}+\ldots\right]_{\mathrm{sym}}$

$+\left\{d_{0}+d_{1} \epsilon+\frac{d_{2}}{2} \epsilon^{2}+\frac{d_{3}}{3} \epsilon^{3}+\frac{d_{4}}{4} \epsilon^{4}+{\frac{d_{5}}{5}}^{5}{ }^{5}+\frac{d_{6}}{6} \epsilon^{6}+\ldots\right\}_{\text {asym }}$

$\Rightarrow \Delta G_{\epsilon}=\frac{1}{2} \Delta G_{x}=d_{1}+\left(c_{2}+d_{2}\right) \epsilon+d_{3} \epsilon^{2}+\left(c_{4}+d_{4}\right) \epsilon^{3}+d_{5} \epsilon^{4}+\left(c_{6}+d_{6}\right) \epsilon^{5}+\ldots$

where

$c_{0}=-k T \ln 2+\frac{v_{2}}{4}+\frac{v_{4}}{16}+\frac{v_{6}}{64}+\ldots$,

$c_{2}=k T-\frac{v_{2}}{2}-\frac{v_{4}}{4}-\frac{3}{32} v_{6}$,

$c_{4}=\frac{1}{3} k T+\frac{v_{4}}{4}+\frac{3 v_{6}}{16}$,

$c_{6}=\frac{1}{5} k T-\frac{3 v_{6}}{32}$,

$d_{0}=\frac{v_{21}}{2^{3}}+\frac{v_{31}}{2^{4}}+\frac{v_{41}}{2^{5}}+\frac{v_{51}}{2^{6}}+\frac{v_{32}}{2^{5}}+\frac{v_{42}}{2^{6}}$

and $d_{i}$ is the coefficient of $\epsilon^{i-1}$ in the asymmetric term of $\Delta G_{\epsilon}$ in braces.

Then, mainly due to the 3-body effect $\left(v_{21}\right)$ which appears in $d_{2}$, the local minimum shifts from the position at $\epsilon=0$ for the symmetric system to $\epsilon=\epsilon_{0} \approx \frac{-d_{1}}{\left(c_{2}+d_{2}\right)}$ for this asymmetrical system, and the double-well minima at $\epsilon=\epsilon_{ \pm}$also shift, turning into one global minimum and one local minimum (which could even disappear) [See Fig. 3(c)]. To have a stable (global) minimum, $c_{6}>0$ and in general $c_{6}>0$ (unless $v_{6} \geq \frac{32}{15} k T$ which is unlikely in most cases).

\section{B. Symmetric $\Delta G$}

To facilitate our discussion, we first focus on the symmetric system up to $6^{\text {th }}$ order where $c_{6}>0$.

$$
\begin{aligned}
& \Delta G=k T x \ln x+k T(1-x) \ln (1-x)+v_{2} x(1-x)+v_{4} x^{2}(1-x)^{2}+v_{6} x^{3}(1-x)^{3} \\
& =c_{0}+1 / 2 c_{2} \epsilon^{2}+1 / 4 c_{4} \epsilon^{4}+1 / 6 c_{6} \epsilon^{6} \\
& \Delta G^{*}=1 / 2 c_{2} \epsilon^{2}+1 / 4 c_{4} \epsilon^{4}+1 / 6 c_{6} \epsilon^{6}
\end{aligned}
$$




$$
\begin{aligned}
& \Delta G_{\epsilon}=\epsilon\left[\left(k T-\frac{v_{2}}{2}-\frac{v_{4}}{4}-\frac{3}{32} v_{6}\right)+\epsilon^{2}\left(\frac{1}{3} k T+\frac{v_{4}}{4}+\frac{3 v_{6}}{16}\right)+\epsilon^{4}\left(\frac{1}{5} k T-\frac{3 v_{6}}{32}\right)\right] \\
& =\epsilon\left[c_{2}+c_{4} \epsilon^{2}+c_{6} \epsilon^{4}\right]=c_{6} \epsilon\left(\epsilon^{2}-\epsilon_{-}{ }^{2}\right)\left(\epsilon^{2}-\epsilon_{+}{ }^{2}\right)
\end{aligned}
$$

where

$\epsilon_{ \pm}^{2}=\left[-c_{4} \pm\left(c_{4}{ }^{2}-4 c_{2} c_{6}\right)^{1 / 2}\right] /\left(2 c_{6}\right)=\frac{-c_{4}}{2 c_{6}} \pm \frac{\left|-c_{4}\right|}{2 c_{6}}\left(1-\frac{4^{c_{2} c_{6}}}{c_{4}{ }^{2}}\right)^{1 / 2}$,

$$
\epsilon_{-} \leq \epsilon_{+} \text {unless } \epsilon_{ \pm} \text {is not imaginary number (i.e., } c_{4}{ }^{2}-4 c_{2} c_{6}<0 \text { ). }
$$

For convenience's sake, we define $f\left(\epsilon^{n}\right)$ as a polynomial function of $\epsilon$ with the highest order $n$ (i.e., $\epsilon^{n}$ ) which has no real root for $f\left(\epsilon^{n}\right)=0$.

Here we consider some possible cases for $\left(c_{2}, c_{4}\right)$ while $c_{6}>0$, as shown in Fig. 3(c).

\section{Phase Transition in symmetric $\Delta G\left(\Delta G^{*}=1 / 2 c_{2} \epsilon^{2}+1 / 4 c_{4} \epsilon^{4}+1 / 6 c_{6} \epsilon^{6}\left(c_{6}>0\right)\right.$}

The order parameter $\epsilon$ corresponds to the $\Delta G_{\min }$ which satisfies

$\Delta G_{\epsilon}=\left.\epsilon\left[c_{2}+c_{4} \epsilon^{2}+c_{6} \epsilon^{4}\right]\right|_{\epsilon= \pm \epsilon_{+}, 0}=0$,

Then we can have equation $6 \mathrm{~b}$ where $\epsilon_{-} \leq \epsilon_{+}$unless $\epsilon_{ \pm}$is not imaginary number (i.e., $c_{4}{ }^{2}-4$ $\left.c_{2} c_{6}<0\right)$ and $\epsilon_{+}$and $\epsilon_{-}$correspond to the minimum and the maximum of $\Delta G$, respectively.

The critical point corresponds to the 2 nd order continuous phase transition where the global minimum (at $\epsilon=0$ ) smoothly splits into two minima at $\left(\epsilon= \pm \epsilon_{+} \neq 0\right)$, while the global minimum changes to a non-stable local maximum. Thus, at $T=T_{c}$, the sign of $c_{2}$ should change. Consequently, the leading term of $c_{2}$ is represented as $c_{2} \propto T-T_{c}: c_{2}=\alpha\left(T-T_{c}\right)$, which can be modified as $c_{2}=\alpha\left(T-T_{c}\right)+$ $\alpha_{2}\left(T-T_{c}\right)^{2}+\cdots$ or $c_{2}=\alpha_{x}\left(T-T_{c}\right)^{x}$, etc., depending on the given system, where $\alpha, \alpha_{2}$ and $\alpha_{x}$ are constants.

If $\Delta G\left(\epsilon=\epsilon_{+}\right)=\Delta G(\epsilon=0)$ with $c_{6}>0$ and $c_{4}<0$, it corresponds to a special first order transition temperature $T_{0}$ that the second order phase changes to the first order.

Despite that the phase transition becomes continuous as second order for $c_{4} \geq 0$, the order parameter $\epsilon$ at the phase transition is discontinuous as first order for $c_{4}<0$ because the global minimum discretely changes from $\epsilon=0$ to $\pm \epsilon_{+}= \pm\left(-\frac{3 c_{4}}{4 c_{6}}\right)^{1 / 2}$.

At temperatures $T_{c}$ and $T_{1}$, by continuous $2^{\text {nd }}$ order transition between phases at $\epsilon=0$ and $\epsilon=\epsilon_{+}$, the heat capacity shows a discontinuity by $\left.\Delta C\right|_{T=T_{c}}=-1 / 2 \frac{\alpha^{2}}{c_{4}} T_{C}$ and $\left.\Delta C\right|_{T=T_{1}}=\infty$ (which diverges), respectively. Therefore, the free energy change $(\Delta G)$ paths with respect to $T$ are different between the case as $T$ changes from low to high temperature (i.e., from the low temperature minimum state to the high temperature minimum state) and the opposite case, showing the hysteresis by the path-dependent heat difference (absorbing/release). Similarly, at temperature $T_{0}$ by discontinuous $1^{\text {st }}$ order transition between phases at $\epsilon=0$ and $\epsilon=\epsilon_{+}$, we find a discontinuity in heat capacity by $\left.\Delta C\right|_{T=T_{0}}=-\frac{\alpha^{2}}{c_{4}}$ $T_{0}$.

\section{Asymmetric $\Delta G$}

Now, let us consider the free energy change of an asymmetric system by including $\frac{c_{3}}{3} \epsilon^{3}$ to that of the symmetric system. In this case, in equation (1-5), the higher order term than fifth order is not so 
important, and so we consider up to the fourth order $\frac{c_{4}}{4} \epsilon^{4}$ in free energy. Then we have:

$$
\begin{aligned}
& \Delta G_{\epsilon}=\epsilon\left[\left(k T-\frac{v_{2}}{2}-\frac{v_{4}}{4}\right)+\epsilon^{2}\left(\frac{1}{3} k T+\frac{v_{4}}{4}\right)\right]_{\mathrm{sym}} \\
& +\left\{\left(\frac{v_{21}}{2^{3}}+\frac{2 v_{31}}{2^{4}}\right)+\epsilon\left(-\frac{v_{21}}{2^{2}}\right)+\epsilon^{2}\left(-\frac{3 v_{21}}{2^{3}}-\frac{3 v_{31}}{2^{3}}\right)+\epsilon^{3}\left(-\frac{v_{31}}{2^{2}}\right)\right\}_{\mathrm{asym}} \\
& =b_{1}+b_{2} \epsilon+b_{3} \epsilon^{2}+b_{4} \epsilon^{3}
\end{aligned}
$$

where

$b_{0}=c_{0}+d_{0}, b_{1}=d_{1}, b_{2}=c_{2}+d_{2}, b_{3}=d_{3}, b_{4}=c_{4}+d_{4}$,

$d_{0}=\frac{v_{21}}{2^{3}}+\frac{v_{31}}{2^{4}}, d_{1}=\frac{v_{21}}{2^{3}}+\frac{2 v_{31}}{2^{4}}, d_{2}=-\frac{v_{21}}{2^{2}}, d_{3}=-\frac{3 v_{21}}{2^{3}}-\frac{3 v_{31}}{2^{3}}, d_{4}=-\frac{v_{31}}{2^{2}}$.

When 2-body and (small but significant) 3-body interactions are mainly considered (if 4-body is rather smaller, and so asymmetric 4-body interactions are neglected), the approximate values of $b_{i}$ coefficients are

$b_{0} \approx-k T \ln 2+\frac{v_{2}}{4}+\frac{v_{4}}{16}+\frac{v_{21}}{2^{3}}, b_{1} \approx \frac{v_{21}}{2^{3}}, b_{2} \approx k T-\frac{v_{2}}{2}-\frac{v_{4}}{4}-\frac{v_{21}}{2^{2}}, b_{3} \approx-\frac{3 v_{21}}{2^{3}}, b_{4} \approx \frac{1}{3} k T+\frac{v_{4}}{4}$,

Then, mainly due to the 3-body effect $\left(v_{21}\right)$ which appears in $d_{2}$, the local minimum at $\epsilon=0$ for the symmetric system shifts to the position at $\epsilon=\epsilon_{0} \approx \frac{-b_{1}}{b_{2}}=\frac{-d_{1}}{\left(c_{2}+d_{2}\right)} \approx \frac{-d_{1}}{c_{2}}\left(\square\left|d_{2}\right| \ll\left|c_{2}\right|\right)$ for this asymmetrical system, and the double-well minima at $\epsilon=\epsilon_{ \pm}$also shift, turning into one global minimum and one local minimum (which could even disappear) [See Fig. 3(c)].

Here we consider some possible cases of $\left(b_{2}, b_{3}\right)$ while $b_{4}>0$, as shown in Fig. 3(d).

At temperatures $T_{c}$ and $T_{1}$, by continuous $2^{\text {nd }}$ order transition between phases at $\epsilon=0$ and $\epsilon=\epsilon_{+}$, the heat capacity gives a discontinuity by $\left.\Delta C\right|_{T=T_{c}}=\frac{4 \alpha^{2}}{b_{4}} T_{C}$ and $\left.\Delta C\right|_{T=T_{1}}=\frac{2 \alpha^{2}}{b_{4}} T_{1}$, respectively, showing the hysteresis with the path-dependent heat difference (absorbing/release). Similarly, at temperature $T_{0}$, by discontinuous $1^{\text {st }}$ order transition between phases at $\epsilon=0$ and $\epsilon=\epsilon_{+}$, the discontinuity in heat capacity is $\left.\Delta C\right|_{T=T_{0}}=\frac{8 \alpha^{2}}{b_{4}} T_{0}$.

\section{E. $\Delta G$ between different states with different chemical potentials}

Here we now include chemical potential change driven $\Delta G^{\mu}$ upon mixing. As discussed earlier in the beginning of this Supplementary Information, the free energy change is given by

$\Delta G=-T \Delta S+\Delta H=-T \Delta S+\Delta H^{i}+\Delta E^{\mu}=\Delta G^{i}+\Delta E^{\mu}=\Delta G^{i}+\bar{\mu}+\frac{\epsilon}{2} \mu_{A B}$,

where

$\Delta E^{\mu}=\bar{\mu}+\frac{\epsilon}{2} \mu_{A B}$.

Let's consider a simple symmetric $\Delta G^{i}=\Delta G^{*}=1 / 2 c_{2} \epsilon^{2}+1 / 4 c_{4} \epsilon^{4}\left(c_{4}>0\right)$. Then,

$\Delta G=\Delta G^{i}+\bar{\mu}+\frac{\mu_{A B}}{2} \epsilon=\bar{\mu}+\frac{\mu_{A B}}{2} \epsilon+1 / 2 c_{2} \epsilon^{2}+1 / 4 c_{4} \epsilon^{4}=c_{0}+c_{1} \epsilon+1 / 2 c_{2} \epsilon^{2}+1 / 4 c_{4} \epsilon^{4}$

where $c_{0}=\bar{\mu} \quad$ and $c_{1}=\frac{\mu_{A B}}{2}$.

Then, $\Delta G_{\epsilon}=c_{1}+c_{2} \epsilon+c_{4} \epsilon^{3}$

This case is already well discussed in Section 4: Free Energy Change in Asymmetric System.

The local minimum at $\epsilon=0$ for the symmetric system $\left(\Delta G^{i}\right)$ has shifted to the position at $\epsilon=\epsilon_{0} \approx$ $\frac{-c_{1}}{c_{2}}$ towards an asymmetrical system, and the double-well minima at $\epsilon=\epsilon_{ \pm}$(for $c_{2}<0$ ) also shift, 
turning into one global minimum and one local minimum (which could even disappear) [See Fig. $3(\mathrm{c})]$.

Note in this symmetric system that at $T=T_{o}$ the two chemical moieties $A$ and $B$ have the same chemical potentials: $\mu_{A}=\mu_{B}$. Depending on $T>T_{o}$ or $T<T_{o}$, the sign of $\mu_{A B}$ changes, and the simple approximation near $T=T_{o}$ is $\mu_{A B} \approx 2 \mu_{o} \gamma\left(T / T_{o}-1\right)$ or $c_{1} \approx \mu_{o} \gamma\left(T / T_{o}-1\right)$ where $\gamma$ is a proportional constant.

In the present confined water systems, two chemical moieties $A$ and $B$ in different phases have the same chemical potentials $\left(\mu_{A B}=c_{1}=0\right)$ at $T_{o}$, but changes the sign of $\mu_{A B}$ or $c_{1}$ depending on $T>T_{o}$ or $T<T_{o}$ because of their difference in rotational degree of freedom depending on $T$.

Setting the mixing-driven free energy change to zero at the equilibrium point $\left(\epsilon=\epsilon_{0} \approx \frac{-c_{1}}{c_{2}}\right)$ where $\left|\epsilon_{0}\right| \approx\left|\frac{c_{1}}{c_{2}}\right|<1 ;\left|\epsilon_{0}\right|^{2} \approx\left|\frac{c_{1}}{c_{2}}\right|^{2} \approx 0 \ll 1$,) or defining $\varepsilon=\epsilon-\epsilon_{0}$, we have the following approximation:

$\Delta G_{\varepsilon} \approx c_{2} \varepsilon-3 \frac{c_{1} c_{4}}{c_{2}} \varepsilon^{2}+c_{4} \epsilon^{3} \approx b_{2} \varepsilon+b_{3} \varepsilon^{2}+b_{4} \varepsilon^{3}$,

or $\Delta G^{*}=1 / 2 b_{2} \epsilon^{2}+1 / 3 b_{3} \epsilon^{4}+1 / 4 b_{4} \epsilon^{4}=b_{4} \varepsilon\left(\varepsilon-\varepsilon_{-}\right)\left(\varepsilon-\varepsilon_{+}\right)$,

where

$\varepsilon_{ \pm}=\left[-b_{3} \pm\left(b_{3}{ }^{2}-4 b_{2} b_{4}\right)^{1 / 2}\right] /\left(2 b_{4}\right)=\frac{-b_{3}}{2 b_{4}} \pm \frac{\left|-b_{3}\right|}{2 b_{4}}\left(1-\frac{4^{b_{2} b_{4}}}{b_{3}{ }^{2}}\right)^{1 / 2}$,

$\varepsilon_{-} \leq \varepsilon_{+}$unless $\varepsilon_{ \pm}$is not imaginary number (i.e., $b_{3}{ }^{2}-4 b_{2} b_{4}<0$ ).

Here, $1 / 3 b_{3} \varepsilon^{3}$, i.e., $-\frac{c_{1} c_{4}}{c_{2}} \varepsilon^{3}$ or $-\left(T-T_{o}\right)_{\frac{\mu_{0} c_{4}}{c_{2}}}^{3} \varepsilon^{3}$ appears as a significant cubic term in $\Delta G^{*}$

for $\left|T-T_{o}\right|>>0$, which results in highly asymmetric potential. If we set $\left|\epsilon_{0}\right| \approx\left|\frac{c_{1}}{c_{2}}\right|<1 ;\left|\epsilon_{0}\right|^{2} \approx\left|\frac{c_{1}}{c_{2}}\right|^{2} \ll 1$;

$\left.\left|\epsilon_{0}\right|^{3} \approx\left|\frac{c_{1}}{c_{2}}\right|^{3} \approx 0 \ll 1\right)$, then the $b_{2}$ term can take into account even the $\left|T-T_{o}\right|^{2}$ term.

If $\Delta G=c_{0}+c_{1} \epsilon+1 / 2 c_{2} \epsilon^{2}+1 / 3 c_{3} \epsilon^{4}+1 / 4 C_{4} \epsilon^{4}$ (where $c_{3}=d_{3}$ :small) is slightly asymmetric at $T=T_{o}$, we will have slightly strengthened asymmetry with $1 / 3\left(b_{3}+d_{3}\right) \varepsilon^{3}=\left[1 / 3 d_{3}-\left(T-T_{o}\right) \frac{\mu_{o} c_{4}}{c_{2}}\right] \varepsilon^{3}$.

All these asymmetric cases are shown in Fig. 3(d). Here we consider all possible cases for all variations of $b_{2}$ and $b_{3}$, while $b_{4}>0$. The behavior is mirror symmetric with respect to the axis of $b_{3}$ (i.e., $\left.b_{2}=0\right)$. Namely, the behaviors for $b_{3}$ and $-b_{3}$ are mirror-symmetric.

\section{F. Mixing-driven enthalpy change due to high order Interactions}

Here we also describe the details of the mixing enthalpy.

Assume that there are $N$ particles comprised of $N_{\mathrm{A}}$ particles of type A and $N_{\mathrm{B}}$ particles of type B (where particles can be replaced by moieties representing each particular phase without losing generality):

$N=N_{\mathrm{A}}+N_{\mathrm{B}}$,

$\mathrm{x}_{\mathrm{A}}=N_{\mathrm{A}} / N, \mathrm{x}_{\mathrm{B}}=N_{\mathrm{B}} / N\left(\right.$ or $\left.\mathrm{x}=\mathrm{x}_{\mathrm{A}}, 1-\mathrm{x}=\mathrm{x}_{\mathrm{B}}\right)$.

Consider the case that each particle of $\mathrm{A}$ has $n_{\mathrm{AA}}$ nearest neighbors by the other particles of $\mathrm{A}$ (i.e., coordination number of $\mathrm{A}$ by $\mathrm{A}$ ) with 2-body interaction energy $v_{\mathrm{AA}}$, while similarly $\mathrm{B}$ has $n_{\mathrm{BB}}$ nearest neighbors by $\mathrm{B}$ with 2-body interaction energy $v_{\mathrm{BB}}$.

Then, the system comprised of the unmixed $N_{\mathrm{A}}$ particles of $\mathrm{A}$ and $N_{\mathrm{B}}$ particles of $\mathrm{B}$ has the 2-body interactions based enthalpy: 
$H_{\text {unmixed }}=1 / 2\left(N_{\mathrm{A}} n_{\mathrm{AA}} v_{\mathrm{AA}}+N_{\mathrm{B}} n_{\mathrm{BB}} v_{\mathrm{BB}}\right)=1 / 2 N\left(\mathrm{x}_{\mathrm{A}} n_{\mathrm{AA}} v_{\mathrm{AA}}+\mathrm{x}_{\mathrm{B}} n_{\mathrm{BB}} v_{\mathrm{BB}}\right)$

where $1 / 2$ is used due to the double counting.

Once $N_{\mathrm{A}} \mathrm{A}$ particles and $N_{\mathrm{B}} \mathrm{B}$ particles are homogeneously mixed, then each particle of A is coordinated by $n_{\mathrm{AB}}$ particles of $\mathrm{B}\left(n_{\mathrm{AB}}\right.$ : coordination number of $\mathrm{A}$ by $\left.\mathrm{B}\right)$ with 2-body $\mathrm{A}-\mathrm{B}$ interaction energy $v_{\mathrm{AB}}$, while similarly $\mathrm{B}$ is coordinated by $n_{\mathrm{BA}}$ particles of $\mathrm{A}$ ( $n_{\mathrm{BA}}$ : coordination number of $\mathrm{B}$ by A) with 2-body interaction energy $v_{\mathrm{BA}}\left(=v_{\mathrm{AB}}\right)$.

The values of $\left(v_{\mathrm{AB}}\right.$ and $\left.n_{\mathrm{AB}}\right)$ in the mixed state are the sums of the linear values $\left(\bar{v}_{\mathrm{AB}}\right.$ and $\left.\bar{n}_{\mathrm{AB}}\right)$ [which will linearly change between the values of two unmixed pure states $\left(v_{\mathrm{AA}}, n_{\mathrm{AA}}\right)$ and $\left.\left(v_{\mathrm{BB}}, n_{\mathrm{BB}}\right)\right]$ and the remaining nonlinear correction values $\left(\Delta v_{\mathrm{AB}}\right.$ and $\left.\Delta n_{\mathrm{AB}}\right)$ :

$v_{\mathrm{AB}}=\bar{v}_{\mathrm{AB}}+\Delta v_{\mathrm{AB}}$,

$\bar{v}_{\mathrm{AB}}=\mathrm{x}_{\mathrm{A}} v_{\mathrm{AA}}+\mathrm{x}_{\mathrm{B}} v_{\mathrm{BB}}$,

$\Delta v_{\mathrm{AB}}=\mathrm{a}_{11} \mathrm{x}_{\mathrm{A}} \mathrm{x}_{\mathrm{B}}+\mathrm{a}_{21} \mathrm{x}_{\mathrm{A}}^{2} \mathrm{x}_{\mathrm{B}}+\mathrm{a}_{12} \mathrm{x}_{\mathrm{A}} \mathrm{x}_{\mathrm{B}}^{2}+\mathrm{a}_{22} \mathrm{x}_{\mathrm{A}}^{2} \mathrm{x}_{\mathrm{B}}^{2}+\ldots \quad$ (a $\mathrm{a}_{\mathrm{ij}}$ : coefficients)

which should satisfy $\Delta v_{\mathrm{AB}}\left(\mathrm{x}_{\mathrm{A}}=1, \mathrm{x}_{\mathrm{B}}=0\right)=\Delta v_{\mathrm{AB}}\left(\mathrm{x}_{\mathrm{A}}=0, \mathrm{x}_{\mathrm{B}}=1\right)=0$.

$\Delta v_{\mathrm{AB}}=\mathrm{a}_{11} \mathrm{x}(1-\mathrm{x})+\mathrm{a}_{21} \mathrm{x}^{2}(1-\mathrm{x})+\mathrm{a}_{12} \mathrm{x}(1-\mathrm{x})^{2}+\mathrm{a}_{22} \mathrm{x}^{2}(1-\mathrm{x})^{2}+\ldots$

$=\left[\mathrm{a}_{11}+\left(\mathrm{a}_{12}+\mathrm{a}_{21}\right) / 2\right] \mathrm{x}(1-\mathrm{x})+\left(\mathrm{a}_{12}-\mathrm{a}_{21}\right) / 2 \mathrm{x}(1-\mathrm{x})(1-2 \mathrm{x})+\mathrm{a}_{22} \mathrm{x}^{2}(1-\mathrm{x})^{2}+\ldots$

If two components $A$ and $B$ are characteristically similar, $a_{21} \approx a_{12}$. Then, we have

$\Delta v_{\mathrm{AB}} \approx\left[\mathrm{a}_{11}+\left(\mathrm{a}_{12}+\mathrm{a}_{21}\right) / 2\right] \mathrm{x}(1-\mathrm{x})+\mathrm{a}_{22} \mathrm{x}^{2}(1-\mathrm{x})^{2}+\ldots$,

which tends to give symmetric higher order corrections.

Similarly,

$n_{\mathrm{AB}}=\bar{n}_{\mathrm{AB}}+\Delta n_{\mathrm{AB}}$,

$\bar{n}_{\mathrm{AB}}=\mathrm{x}_{\mathrm{A}} n_{\mathrm{AA}}+\mathrm{x}_{\mathrm{B}} n_{\mathrm{BB}}$,

$\Delta n_{\mathrm{AB}}=\mathrm{s}_{11} \mathrm{x}_{\mathrm{A}} \mathrm{x}_{\mathrm{B}}+\mathrm{s}_{21} \mathrm{x}_{\mathrm{A}}^{2} \mathrm{x}_{\mathrm{B}}+\mathrm{s}_{12} \mathrm{x}_{\mathrm{A}} \mathrm{x}_{\mathrm{B}}^{2}+\mathrm{s}_{22} \mathrm{x}_{\mathrm{A}}{ }^{2} \mathrm{x}_{\mathrm{B}}^{2}+\ldots$ ( $\mathrm{s}_{\mathrm{ij}}$ : coefficients)

$=\mathrm{s}_{11} \mathrm{x}(1-\mathrm{x})+\mathrm{s}_{21} \mathrm{x}^{2}(1-\mathrm{x})+\mathrm{s}_{12} \mathrm{x}(1-\mathrm{x})^{2}+\mathrm{s}_{22} \mathrm{x}^{2}(1-\mathrm{x})^{2}+\ldots$

$=\left[\mathrm{s}_{11}+\left(\mathrm{s}_{12}+\mathrm{s}_{21}\right) / 2\right] \mathrm{x}(1-\mathrm{x})+\left(\mathrm{s}_{12}-\mathrm{s}_{21}\right) / 2 \mathrm{x}(1-\mathrm{x})(1-2 \mathrm{x})+\mathrm{s}_{22} \mathrm{x}^{2}(1-\mathrm{x})^{2}+\ldots$,

$\approx\left[\mathrm{s}_{11}+\left(\mathrm{s}_{12}+\mathrm{s}_{21}\right) / 2\right] \mathrm{x}(1-\mathrm{x})+\mathrm{s}_{22} \mathrm{x}^{2}(1-\mathrm{x})^{2}+\ldots$ (for two similar components $\mathrm{A}$ and $\left.\mathrm{B}: \mathrm{s}_{12} \approx \mathrm{s}_{21}\right)$,

If $\Delta v_{\mathrm{AB}}$ is negative, it indicates strong $\mathrm{A}-\mathrm{B}$ binding, which reduces the $\mathrm{A}-\mathrm{B}$ distance. Then, the $\Delta n$ ${ }_{\mathrm{AB}}$ tends to be negative. If $v_{\mathrm{AB}}$ is negative while $n_{\mathrm{AB}}$ is positive, their product $n_{\mathrm{AB}} v_{\mathrm{AB}}$ tends to cancel somewhat partially, but it still tends to deviate from the linear relationship.

$n_{\mathrm{AB}} v_{\mathrm{AB}}=\bar{n}_{\mathrm{AB}} \bar{v}_{\mathrm{AB}}+\Delta\left(n_{\mathrm{AB}} v_{\mathrm{AB}}\right)$,

$\bar{n}_{\mathrm{AB}} \bar{v}_{\mathrm{AB}}=\mathrm{x}_{\mathrm{A}} n_{\mathrm{AA}} v_{\mathrm{AB}}+\mathrm{x}_{\mathrm{B}} n_{\mathrm{BB}} v_{\mathrm{AB}}$

$\Delta\left(n_{\mathrm{AB}} v_{\mathrm{AB}}\right)=\mathrm{t}_{11} \mathrm{x}_{\mathrm{A}} \mathrm{x}_{\mathrm{B}}+\mathrm{t}_{21} \mathrm{x}_{\mathrm{A}}^{2} \mathrm{x}_{\mathrm{B}}+\mathrm{t}_{12} \mathrm{x}_{\mathrm{A}} \mathrm{x}_{\mathrm{B}}^{2}+\mathrm{t}_{22} \mathrm{x}_{\mathrm{A}}{ }^{2} \mathrm{x}_{\mathrm{B}}{ }^{2}+\ldots \quad\left(\mathrm{t}_{\mathrm{ij}}:\right.$ coefficients $)$

$=\mathrm{t}_{11} \mathrm{x}(1-\mathrm{x})+\mathrm{t}_{21} \mathrm{x}^{2}(1-\mathrm{x})+\mathrm{t}_{12} \mathrm{x}(1-\mathrm{x})^{2}+\mathrm{t}_{22} \mathrm{x}^{2}(1-\mathrm{x})^{2}+\ldots$

$=\left[\mathrm{t}_{11}+\left(\mathrm{t}_{12}+\mathrm{t}_{21}\right) / 2\right] \mathrm{x}(1-\mathrm{x})+\left(\mathrm{t}_{12}-\mathrm{t}_{21}\right) / 2 \mathrm{x}(1-\mathrm{x})(1-2 \mathrm{x})+\mathrm{t}_{22} \mathrm{x}^{2}(1-\mathrm{x})^{2}+\ldots$,

$\approx\left[\mathrm{t}_{11}+\left(\mathrm{t}_{12}+\mathrm{t}_{21}\right) / 2\right] \mathrm{x}(1-\mathrm{x})+\mathrm{t}_{22} \mathrm{x}^{2}(1-\mathrm{x})^{2}+\ldots$ (for two similar components $\mathrm{A}$ and $\left.\mathrm{B}: \mathrm{t}_{12} \approx \mathrm{t}_{21}\right)$,

which tends to give symmetric higher order corrections.

The enthalpy of the mixed state due to 2-body interactions (where each A tends to have the coordination numbers of $\mathrm{x}_{\mathrm{A}} n_{\mathrm{AA}}$ for the neighboring A's and $\mathrm{x}_{\mathrm{B}} n_{\mathrm{AB}}$ for the neighboring $\mathrm{B}$ 's, while each $B$ tends to have in the same way) is 
$H_{\text {mixed }}=1 / 2\left[N_{\mathrm{A}}\left(\mathrm{x}_{\mathrm{A}} n_{\mathrm{AA}} v_{\mathrm{AA}}+\mathrm{x}_{\mathrm{B}} n_{\mathrm{AB}} v_{\mathrm{AB}}\right)+N_{B}\left(\mathrm{x}_{\mathrm{B}} n_{\mathrm{BB}} v_{\mathrm{BB}}+\mathrm{x}_{\mathrm{A}} n_{\mathrm{BA}} v_{\mathrm{BA}}\right)\right]$

$=1 / 2 N\left[\left(\mathrm{x}_{\mathrm{A}}^{2} n_{\mathrm{AA}} v_{\mathrm{AA}}+\mathrm{x}_{\mathrm{A}} \mathrm{x}_{\mathrm{B}} \mathrm{n}_{\mathrm{AB}} v_{\mathrm{AB}}\right)+\left(\mathrm{x}_{\mathrm{B}}^{2} \mathrm{n}_{\mathrm{BB}} v_{\mathrm{BB}}+\mathrm{x}_{\mathrm{A}} \mathrm{x}_{\mathrm{B}} \mathrm{n}_{\mathrm{BA}} v_{\mathrm{BA}}\right)\right]$

$=1 / 2 N\left[\mathrm{x}_{\mathrm{A}} \mathrm{x}_{\mathrm{B}}\left(n_{\mathrm{AB}} v_{\mathrm{AB}}+n_{\mathrm{BA}} v_{\mathrm{BA}}\right)+\left(\mathrm{x}_{\mathrm{A}}^{2} n_{\mathrm{AA}} v_{\mathrm{AA}}+\mathrm{x}_{\mathrm{B}}^{2} n_{\mathrm{BB}} v_{\mathrm{BB}}\right)\right]$

Then, the enthalpy change by mixing from the unmixed state is

$\Delta H_{\text {mixing }}=H_{\text {mixed }}-H_{\text {unmixed }}=1 / 2 N \mathrm{x}_{\mathrm{A}} \mathrm{x}_{\mathrm{B}}\left[\left(n_{\mathrm{AB}} v_{\mathrm{AB}}+n_{\mathrm{BA}} v_{\mathrm{BA}}\right)-\left(n_{\mathrm{AA}} v_{\mathrm{AA}}+n_{\mathrm{BB}} v_{\mathrm{BB}}\right)\right]$,

The enthalpy change per mol due to mixing is

$\Delta H_{\text {mixing }}=1 / 2 \mathrm{x}_{\mathrm{A}} \mathrm{x}_{\mathrm{B}}\left[\left(n_{\mathrm{AB}} v_{\mathrm{AB}}+n_{\mathrm{BA}} v_{\mathrm{BA}}\right)-\left(n_{\mathrm{AA}} v_{\mathrm{AA}}+n_{\mathrm{BB}} v_{\mathrm{BB}}\right)\right]$

$=1 / 2 \times(1-\mathrm{x})\left[\left(n_{\mathrm{AB}} v_{\mathrm{AB}}+n_{\mathrm{BA}} v_{\mathrm{BA}}\right)-\left(n_{\mathrm{AA}} v_{\mathrm{AA}}+n_{\mathrm{BB}} v_{\mathrm{BB}}\right)\right]$.

$\Delta H_{\text {mixing }}=\Sigma_{\mathrm{AB}}\left[\mathrm{x}_{\mathrm{A}} \mathrm{x}_{\mathrm{B}} \hat{n}_{\mathrm{AB}} v_{\mathrm{AB}}\right]$,

where $\hat{n}_{\mathrm{AB}}=n_{\mathrm{AB}}$ and $\hat{n}_{\mathrm{BA}}=n_{\mathrm{BA}}$ for the unmixed pure states

and $\hat{n}_{\mathrm{AA}}=-n_{\mathrm{AA}}$ and $\hat{n}_{\mathrm{BB}}=-n_{\mathrm{BB}}$ for the unmixed pure states.

If $\mathrm{A} \neq \mathrm{B}, \mathrm{x}_{\mathrm{A}}=\mathrm{x}, \mathrm{x}_{\mathrm{B}}=1-\mathrm{x}$, and $\Delta H_{\text {mixing }}=\Sigma_{\mathrm{AB}}\left[\mathrm{x}(1-\mathrm{x}) \hat{n}_{\mathrm{AB}} v_{\mathrm{AB}}\right]$.

If $\mathrm{A}=\mathrm{B}, \mathrm{x}_{\mathrm{A}}=1, \mathrm{x}_{\mathrm{B}}=0$, and $\Delta H_{\text {mixing }}=0$.

Similarly, the enthalpy change by the effective three-body interaction term $\left(v_{\mathrm{ABC}}\right)$ for the number of effective 3-body triples $\left(n_{\mathrm{ABC}}\right)$ due to mixing of types $\mathrm{A}, \mathrm{B}$, and $\mathrm{C}$ is

$\Delta H_{\text {mixing }}=\Sigma_{\mathrm{ABC}} \mathrm{x}_{\mathrm{A}} \mathrm{x}_{\mathrm{B}} \mathrm{x}_{\mathrm{C}} \hat{n}_{\mathrm{ABC}} v_{\mathrm{ABC}}$,

where $\hat{n}_{\mathrm{ABC}}$ shows signs similar to the aforementioned case ( + with respect to $n_{\mathrm{ABC}}$ for the mixed states, but - for the unmixed pure states).

If $\mathrm{B}=\mathrm{C} \neq \mathrm{A}, \mathrm{x}_{\mathrm{A}}=\mathrm{x}$ and $\mathrm{x}_{\mathrm{B}}=\mathrm{x}_{\mathrm{C}}=1-\mathrm{x}$, then equation (15) gives

$\Delta H_{\text {mixing }}=\Sigma_{\mathrm{AB}} \mathrm{x}(1-\mathrm{x})^{2} \hat{n}_{\mathrm{ABB}} v_{\mathrm{ABB}}$.

However, if only additive effective two-body interactions are considered for the whole system, nonadditive many body interactions (such as $v_{12}$ etc.) cannot be taken into account. Nevertheless, there exist higher order interaction terms, as in the following.

In the above derivation, if we consider that $n_{\mathrm{AB}} v_{\mathrm{AB}}=\bar{n}_{\mathrm{AB}} \bar{v}_{\mathrm{AB}}+\Delta\left(n_{\mathrm{AB}} v_{\mathrm{AB}}\right)$ with equations $(21,22)$, the indirect many-body effect in $\Delta H_{\text {mixing }}\left(=\Sigma_{\mathrm{AB}}\left[\mathrm{x}_{\mathrm{A}} \mathrm{x}_{\mathrm{B}} \hat{n}_{\mathrm{AB}} v_{\mathrm{AB}}\right]\right)$ (i.e., the effective two body interactions by the influence of other many particles involved in only two body interactions) appears as nearly symmetrical higher order corrections.

When we also include the second nearest neighbor interactions (in addition to the first nearest neighbor interactions derived above), the derivation can actually be done in the same way, and thus, the above derivation holds more broadly. 


\section{Tables}

Table S1. Temperature dependent free energy change profiles for symmetric

$\Delta G=k T x \ln x+k T(1-x) \ln (1-x)+v_{2} x(1-x)+v_{4} x^{2}(1-x)^{2}+v_{6} x^{3}(1-x)^{3}$, $\Delta G^{*}=1 / 2 c_{2} \epsilon^{2}+1 / 4 c_{4} \epsilon^{4}+1 / 6 c_{6} \epsilon^{6}\left(\right.$ where $c_{6}>0, x=\frac{1}{2}+\frac{\epsilon}{2}$ and $\left.\left.c_{2}=k\left(T-T_{c}\right)\right) ; \Delta G^{*}=\left.\Delta G\right|_{c_{0}=0}\right)$.

\begin{tabular}{|c|c|c|c|c|c|c|}
\hline temperature & Case & $\begin{array}{c}\text { Real roots by } \\
\text { factorization } \\
\text { of quantic } \\
\text { polynomial } G_{\epsilon} / \\
c_{6}\end{array}$ & $\epsilon=0$ & $\epsilon^{2}=\epsilon_{-}{ }^{2}$ & $\epsilon^{2}=\epsilon_{+}{ }^{2}$ & note \\
\hline $\begin{array}{c}T=T_{t} \\
\underset{\text { tri-critical }}{\text { point }}\end{array}$ & $\begin{array}{l}c_{2}=0 \\
c_{4}=0\end{array}$ & $\epsilon^{5}$ & $\begin{array}{l}\Delta G_{\epsilon \epsilon}>0 \\
\text { quintuple } \\
\text { roots: min }\end{array}$ & $\epsilon_{-}{ }^{2}<0$ & $\epsilon_{+}{ }^{2}<0$ & $\begin{array}{c}\text { First-order } \\
\text { transition (at } \boldsymbol{T}_{\mathbf{0}} \\
\left.\text { for } c_{4}<0\right) \\
\text { meets second- } \\
\text { order transition } \\
\text { (at } \boldsymbol{T}=\boldsymbol{T}_{\boldsymbol{c}} \text { for } c_{4} \\
\quad>0 \text { ) }\end{array}$ \\
\hline$T_{C}$ & $\begin{array}{l}c_{2}=0 \\
c_{4}>0\end{array}$ & $\epsilon^{3} f\left(\epsilon^{2}\right)$ & $\begin{array}{c}\Delta G_{\epsilon \epsilon}=0 \\
\text { triple roots; } \\
\quad \text { min }\end{array}$ & $\epsilon_{-}{ }^{2}=0$ & $\epsilon_{+}{ }^{2}<0$ & $\begin{array}{l}\text { critical } \\
\text { temperature }\end{array}$ \\
\hline$T>T_{1}$ & $\begin{array}{c}c_{2}>0 \\
c_{4}>-\left(4 c_{2} c_{6}\right)\end{array}$ & $\epsilon f\left(\epsilon^{4}\right)$ & $\begin{array}{c}\Delta G_{\epsilon \epsilon}>0 \\
\text { single root; } \\
\quad \text { min }\end{array}$ & $\epsilon_{-}{ }^{2}<0$ & $\epsilon_{+}{ }^{2}<0$ & $\begin{array}{l}\text { no phase } \\
\text { transition }\end{array}$ \\
\hline $\begin{array}{c}T_{1} \\
\text { (2nd order } \\
\text { phase } \\
\text { transition) }\end{array}$ & $\begin{array}{c}c_{2}=\frac{c_{4}^{2}}{4 c_{6}} \\
c_{4}<0\end{array}$ & $\begin{array}{c}\epsilon\left(\epsilon^{2}-\epsilon_{+}{ }^{2}\right)^{2} \\
=\epsilon\left(\epsilon-\epsilon_{+}\right)^{2}( \\
\left.\epsilon+\epsilon_{+}\right)^{2} \\
\pm \epsilon_{+}: \text {double } \\
\text { roots }\end{array}$ & $\begin{array}{c}\Delta G_{\epsilon \epsilon}>0 \\
\min \end{array}$ & $\epsilon_{-}{ }^{2}=\epsilon_{+}{ }^{2}=$ & $\begin{array}{c}\Delta G_{\epsilon \epsilon}=0 \\
\text { inflection } \\
\text { points }\end{array}$ & $\begin{array}{c}\text { two inflection } \\
\text { points of double } \\
\text { roots at } \epsilon_{+} \\
\left(=\epsilon_{-}\right) \text {and one } \\
\min \text { at } \epsilon=0 . \\
{\left[T=T_{1}\right]}\end{array}$ \\
\hline $\begin{array}{c}T_{0} \\
\text { (1st order } \\
\text { phase } \\
\text { transition) }\end{array}$ & $\begin{array}{c}c_{2}=\frac{3 c_{4}^{2}}{16 c_{6}} \\
c_{4}<0\end{array}$ & $\begin{array}{c}\epsilon\left(\epsilon^{2}-\epsilon_{-}{ }^{2}\right) \\
\square\left(\epsilon^{2}-\epsilon_{+}{ }^{2}\right) \\
\Delta G(\epsilon=0) \\
=\Delta G \\
\left(\epsilon= \pm \epsilon_{+}\right)\end{array}$ & $\begin{array}{c}\Delta G_{\epsilon \epsilon}>0 \\
\min \end{array}$ & $\begin{array}{c}\Delta G_{\epsilon \epsilon}<0 \\
\text { two } \max \end{array}$ & $\Delta G_{\epsilon \epsilon}>0: \min$ & $\begin{array}{l}\text { First-order phase } \\
\text { transition }\left[T=T_{0}\right]\end{array}$ \\
\hline $\begin{array}{c}T_{C} \\
\text { (2nd order } \\
\text { phase } \\
\text { transition) }\end{array}$ & $\begin{array}{l}c_{2}=0 \\
c_{4}<0\end{array}$ & $\begin{array}{c}\epsilon^{3}\left(\epsilon^{2}-\epsilon_{+}{ }^{2}\right) \\
=\epsilon^{3}\left(\epsilon-\epsilon_{+}\right) \\
\left(\epsilon+\epsilon_{+}\right)\end{array}$ & $\begin{array}{l}\Delta G_{\epsilon \epsilon}=0 \\
\text { triple roots; } \\
\quad \max \end{array}$ & $\epsilon_{-}^{2}=0$ & $\begin{array}{c}\epsilon_{+}{ }^{2}=-\frac{c_{4}}{c_{6}}>0 \\
\Delta G_{\epsilon \epsilon}>0: \\
\text { two min }\end{array}$ & $\begin{array}{c}\text { meta-stable } \\
\text { critical } \\
\text { temperature at } \\
\boldsymbol{T}=\boldsymbol{T}_{\boldsymbol{c}} \\
\end{array}$ \\
\hline
\end{tabular}

$\frac{\Delta G_{x}}{2}=\Delta G_{\epsilon}=c_{2}+c_{4} \epsilon^{2}+c_{6} \epsilon^{4}=c_{6} \epsilon\left(\epsilon^{2}-\epsilon_{-}^{2}\right)\left(\epsilon^{2}-\epsilon_{+}{ }^{2}\right)$,

where $\epsilon_{ \pm}{ }^{2}=\frac{\left|c_{4}\right|}{2 c_{6}}\left[1 \pm\left(1-\frac{c_{2} c_{6}}{c_{4}{ }^{2}}\right)^{1 / 2}\right]\left(\epsilon_{+} \geq \epsilon_{-} \geq 0\right.$ when $\left.c_{4}^{2} \geq 4 c_{2} c_{6}\right)$.

$\Delta G_{\epsilon \epsilon} \equiv \frac{\partial G_{\epsilon}}{\partial \epsilon} . f\left(\epsilon^{n}\right)$ is the $\mathrm{n}^{\text {th }}$ order function of $\epsilon$ without real roots [see Fig. 3(c)]. 
Table S2. Temperature dependent free energy change profiles for asymmetric $\Delta G^{*}=1 / 2 b_{2} \varepsilon^{2}+1 / 3 b_{6} \varepsilon^{3}+1 / 4 b_{4} \varepsilon^{4}$ (where $b_{4}>0, x=\frac{1}{2}+\frac{\varepsilon}{2}$ and $c_{2}=k\left(T-T_{c}\right.$ ); mirror images with respect to the $b_{2}$ axis (i.e., $b_{3}=0$ ); thus, the profile is discussed only for $b_{3} \leq 0$ ).

\begin{tabular}{|c|c|c|c|c|c|c|}
\hline temperature & Case & $\begin{array}{c}\text { Real roots by } \\
\text { factorization } \\
\text { of cubic } \\
\text { polynomial } G_{\epsilon} / \\
b_{4}\end{array}$ & $\epsilon=0$ & $\boldsymbol{\epsilon}_{-}$ & $\boldsymbol{\epsilon}_{+}$ & note \\
\hline$T=T_{t}$ & $\begin{array}{l}b_{2}=0 \\
b_{3}=0\end{array}$ & $\epsilon^{3}$ & $\begin{array}{l}\Delta G_{\epsilon \epsilon}>0 \\
\text { triple min }\end{array}$ & & & $\begin{array}{c}\text { Tri-critical point } \\
\text { at } T=T_{t} \text { where } \\
2^{\text {nd }} \text { order meta- } \\
\text { stable transitions } \\
\text { meets } 1^{\text {st }} \text { order } \\
\text { transition at } b_{3} \\
=0 \\
\text { for } T=T_{0}\end{array}$ \\
\hline$T<T_{C}$ & $\begin{array}{r}b_{2}<0, \\
b_{3}<0\end{array}$ & $\begin{array}{c}\epsilon\left(\epsilon-\epsilon_{+}\right) \\
\square\left(\epsilon+\epsilon_{+}\right) \\
\epsilon_{-}<0<\epsilon_{+}\end{array}$ & $\begin{array}{l}\Delta G_{\epsilon \epsilon}<0 \\
\text { local max }\end{array}$ & $\begin{array}{l}\Delta G_{\epsilon \epsilon}>0: \\
\text { local min }\end{array}$ & $\begin{array}{l}\Delta G_{\epsilon \epsilon}>0: \\
\text { global min }\end{array}$ & \\
\hline$T_{C}$ & $\begin{array}{c}b_{2}<0 \\
b_{3}=0\end{array}$ & $\begin{array}{c}\epsilon\left(\epsilon-\epsilon_{-}\right) \\
\square\left(\epsilon-\epsilon_{+}\right) \\
\varepsilon_{+}=\varepsilon_{-}>0 \\
\left.\Delta G^{*}\right|_{\varepsilon=\varepsilon_{+}}= \\
\left.\Delta G^{*}\right|_{\varepsilon=\varepsilon_{-}}\end{array}$ & $\begin{array}{l}\Delta G_{\epsilon \epsilon}=0 \\
\text { local max }\end{array}$ & $\begin{array}{c}\varepsilon_{-}=-\left(\frac{-b_{2}}{b_{4}}\right. \\
)^{\frac{1}{2}} \\
\text { min }\end{array}$ & $\begin{aligned} \varepsilon_{+} & =\left(\frac{-b_{2}}{b_{4}}\right)^{\frac{1}{2}} \\
& \min \end{aligned}$ & \\
\hline$T>T_{1}$ & $\begin{array}{c}b_{2}>\frac{b_{3}{ }^{2}}{4 b_{4}} \\
b_{3}<0\end{array}$ & $\epsilon f\left(\epsilon^{2}\right)$ & $\begin{array}{c}\Delta G_{\epsilon \epsilon}>0 \\
\text { single root: } \\
\min \end{array}$ & & & \\
\hline $\begin{array}{c}T_{1} \\
\text { (2nd order } \\
\text { phase } \\
\text { transition) }\end{array}$ & $\begin{array}{c}b_{2}=\frac{b_{3}^{2}}{4 b_{4}} \\
b_{3}<0\end{array}$ & $\begin{array}{l}\epsilon\left(\epsilon-\epsilon_{+}\right)^{2} \\
\epsilon_{+}: \text {double } \\
\text { roots }\end{array}$ & $\begin{array}{c}\Delta G_{\epsilon \epsilon}>0 \\
\min \end{array}$ & $\epsilon_{-}=\epsilon_{+}=-$ & $\begin{array}{c}\Delta G_{\epsilon \epsilon}=0 \\
\text { inflection } \\
\text { point }\end{array}$ & \\
\hline$T_{0}<T<T_{1}$ & $\begin{array}{c}\frac{2 b_{3}^{2}}{9 b_{4}}<b_{2}<\frac{b_{3}}{4 b} \\
b_{3}<0\end{array}$ & $\begin{array}{c}\epsilon\left(\epsilon-\epsilon_{-}\right) \\
\square\left(\epsilon-\epsilon_{+}\right) \\
0 \leq \epsilon_{-} \leq \epsilon_{+}\end{array}$ & $\begin{array}{c}\Delta G_{\epsilon \epsilon}>0 \\
\text { global min }\end{array}$ & $\begin{array}{l}\Delta G_{\epsilon \epsilon}<0 \\
\text { local max }\end{array}$ & $\begin{array}{c}\Delta G_{\epsilon \epsilon} 0 \\
\text { local min }\end{array}$ & \\
\hline $\begin{array}{c}T_{0} \\
\text { (1st order } \\
\text { phase } \\
\text { transition) }\end{array}$ & $\begin{array}{c}b_{2}=\frac{2 b_{3}^{2}}{9 b_{4}}, b_{3} \\
<0\end{array}$ & $\begin{array}{c}\epsilon\left(\epsilon-\epsilon_{-}\right) \\
\square\left(\epsilon-\epsilon_{+}\right) \\
0 \leq \epsilon-\epsilon_{+} \\
\Delta G(\epsilon=0) \\
=\Delta G \\
\left(\epsilon= \pm \epsilon_{+}\right)\end{array}$ & $\begin{array}{c}\Delta G_{\epsilon \epsilon}>0 \\
\text { degenerate } \\
\text { min }\end{array}$ & $\begin{array}{l}\Delta G_{\epsilon \epsilon}<0 \\
\text { local max }\end{array}$ & $\begin{array}{c}\Delta G_{\epsilon \epsilon}>0: \\
\text { degenerate } \\
\quad \text { min }\end{array}$ & \\
\hline$T_{C}<T<T_{0}$ & $\begin{array}{c}0<b_{2}<\frac{2 b_{3}^{2}}{9 b_{4}} \\
b_{3}<0\end{array}$ & $\begin{array}{c}\epsilon\left(\epsilon-\epsilon_{-}\right) \\
\left(\epsilon-\epsilon_{+}\right) \\
0 \leq \epsilon_{-} \leq \epsilon_{+}\end{array}$ & $\begin{array}{l}\Delta G_{\epsilon \epsilon}>0 \\
\text { local min }\end{array}$ & $\begin{array}{c}\Delta G_{\epsilon \epsilon}<0 \\
\text { local max }\end{array}$ & $\begin{array}{c}\Delta G_{\epsilon \epsilon}>0 \\
\text { global min }\end{array}$ & \\
\hline $\begin{array}{c}T_{C} \\
\text { (2nd order } \\
\text { phase } \\
\text { transition) }\end{array}$ & $\begin{array}{c}b_{2}=0 \\
b_{3}<0\end{array}$ & $\begin{array}{c}\epsilon^{2}\left(\epsilon+\frac{b_{3}}{b_{4}}\right) \\
=\epsilon^{2}\left(\epsilon-\epsilon_{+}\right. \\
)\end{array}$ & $\begin{array}{c}\Delta G_{\epsilon \epsilon}=0 \\
\text { inflection } \\
\text { point }\end{array}$ & & $\begin{array}{c}\epsilon_{+}=-\frac{b_{3}}{b_{4}}>0 \\
\Delta G_{\epsilon \epsilon}>0: \\
\min \end{array}$ & \\
\hline
\end{tabular}


(a)

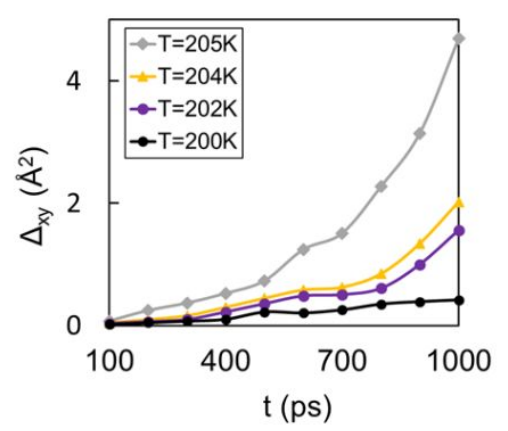

(b)

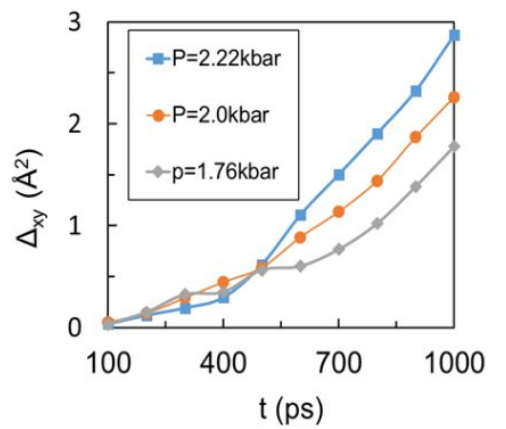

(c)

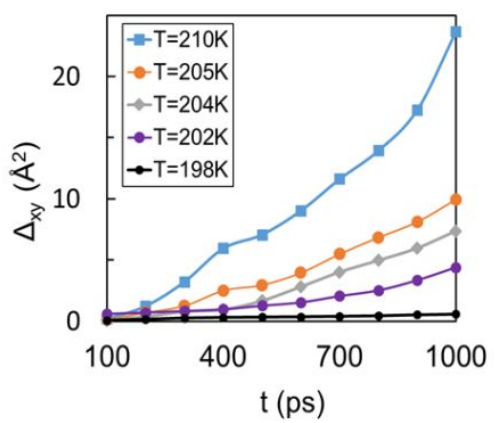

Figure S1: Time evolution of the lateral-MSD $\left(\Delta_{\mathrm{xy}}(\mathrm{t})=<\left[\mathrm{r}_{\mathrm{xy}}(\mathrm{t})-\mathrm{r}_{\mathrm{xy}}(0)\right]^{2}>\right)$ for the confined water. (a) $N V T$ simulations on confined water $\rho=1.05 \mathrm{~g} / \mathrm{cm}^{3}$. (b) $N P_{L} T$ simulations under positive lateralpressure at $T=204 \mathrm{~K}$. (c) $N V T$ simulations on confined water $\rho=0.965 \mathrm{~g} / \mathrm{cm}^{3}$.
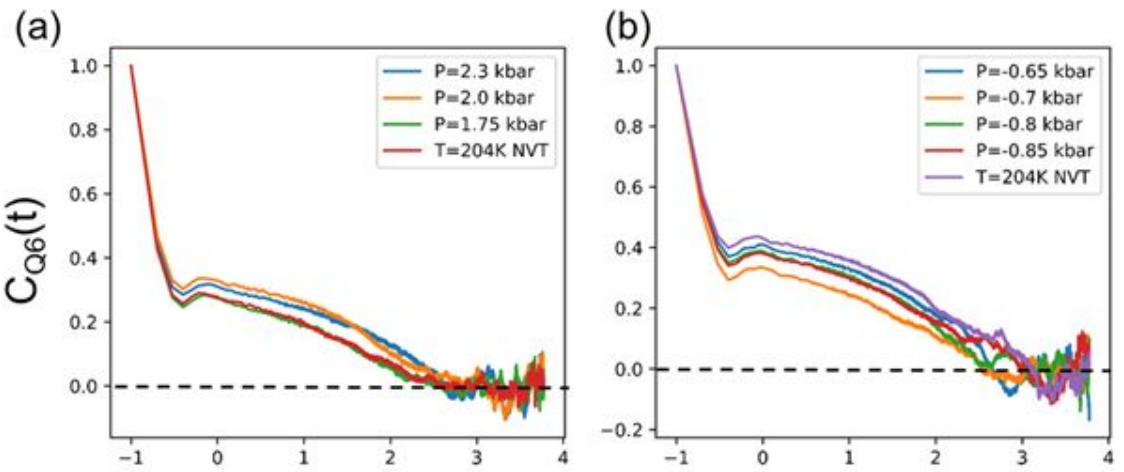

(c)

(d)
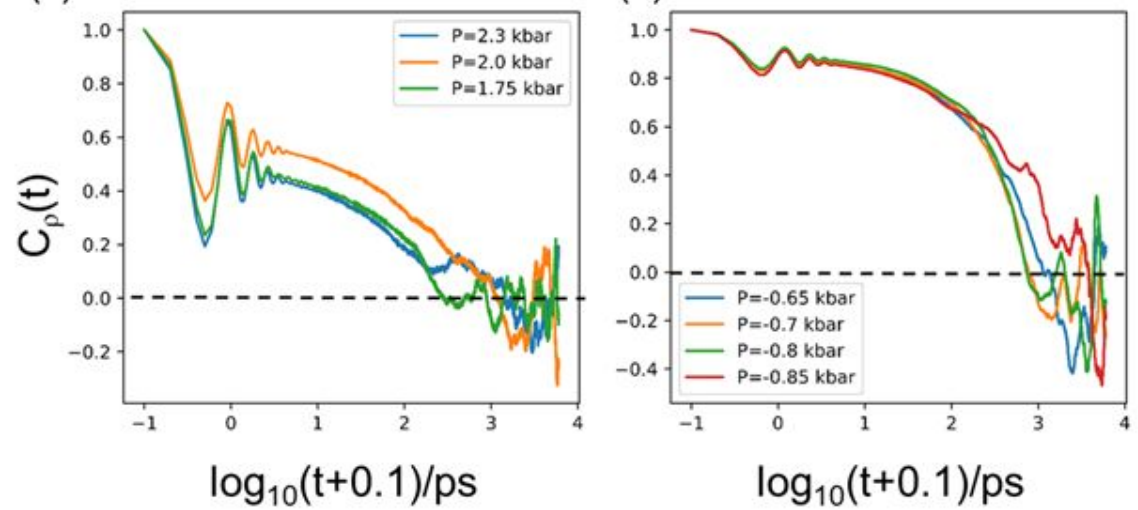

Figure S2: Autocorrelation functions for $Q_{6}(\mathrm{a}, \mathrm{b})$ with $N P_{L} T$ simulation at $T=204 \mathrm{~K}$ under different positive and negative lateral-pressures and Autocorrelation functions for density $(\mathrm{c}, \mathrm{d})$ at the same temperature and different lateral pressures. 


\section{Structural analysis of the states:}

(a)

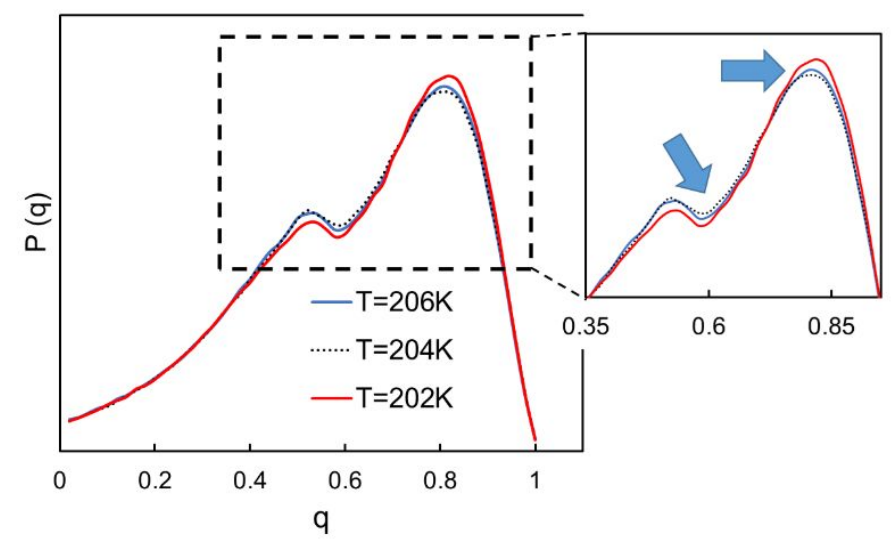

(b)

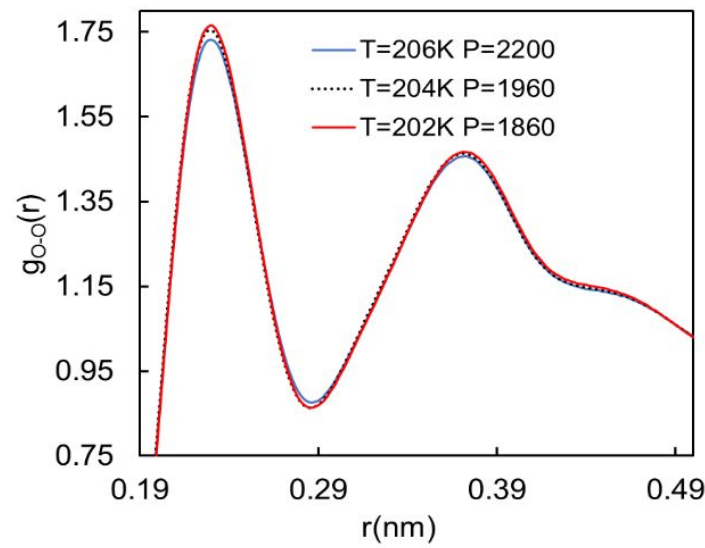

(c)

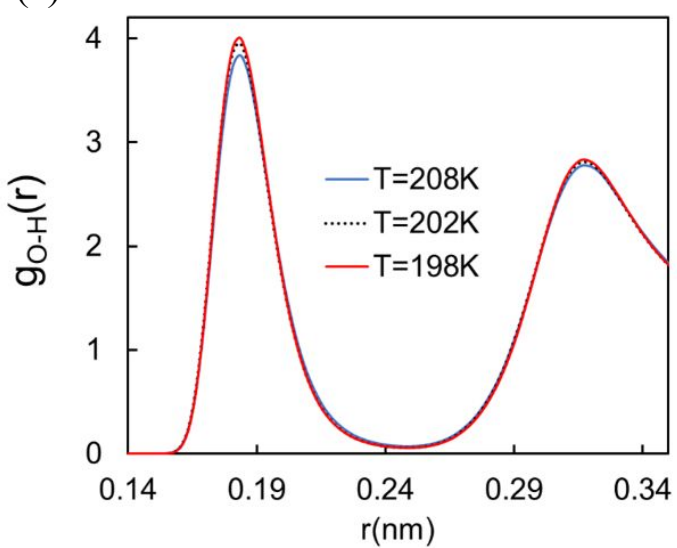

Figure S3: Structural analysis of the states, (a) Probability distribution for the tetrahedral order parameter for confined water with $\rho=1.05 \mathrm{~g} / \mathrm{cm}^{3}$, LDL $(\mathrm{T}=202 \mathrm{~K})$ is more and MDL $(\mathrm{T}=204 \mathrm{~K})$ is less ordered (b) Oxygen-oxygen radial distribution function (RDF) for confined water under positive pressure and (c) O-H RDF under negative pressure.

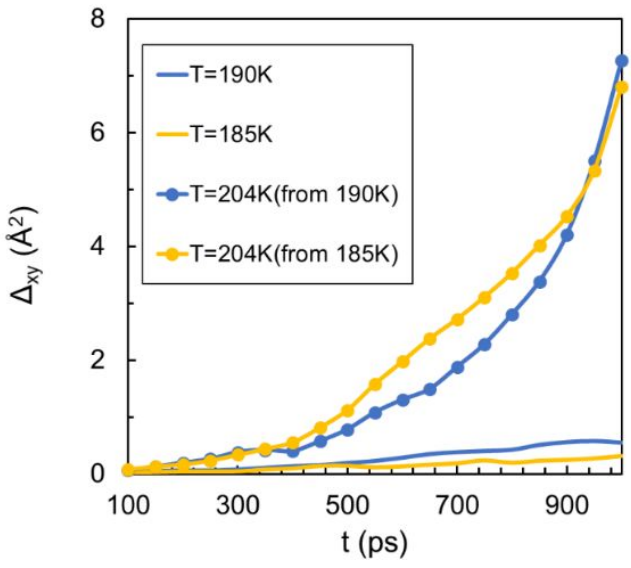

Figure S4: $N V T$ simulations for the confined water $\left(\rho=0.965 \mathrm{~g} / \mathrm{cm}^{3}\right)$ which were initiated from two different configurations of the ice phase at $T=190$ and $185 \mathrm{~K}$. The positive slope of the lateral-MSD shows that the intermediate state from $N V T$ simulation on confined water is different from the ice state and it shows liquid properties. 
(a)

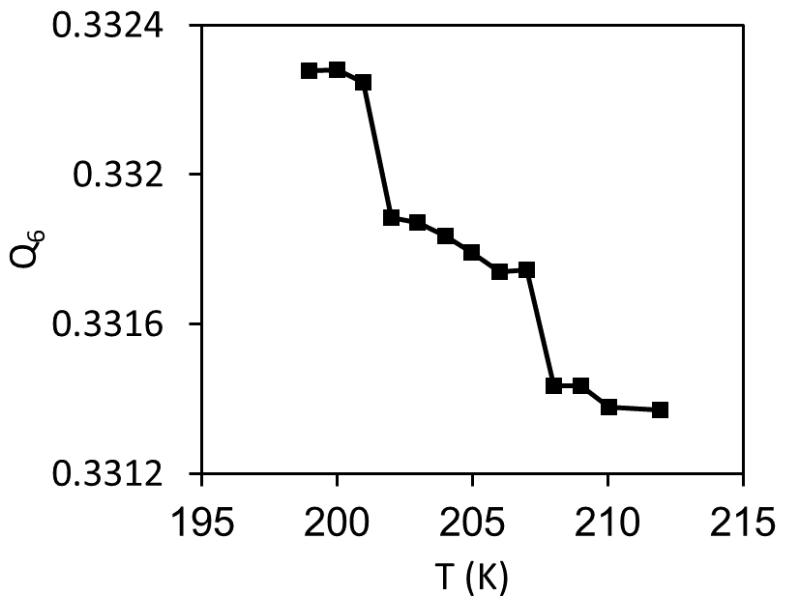

(b)

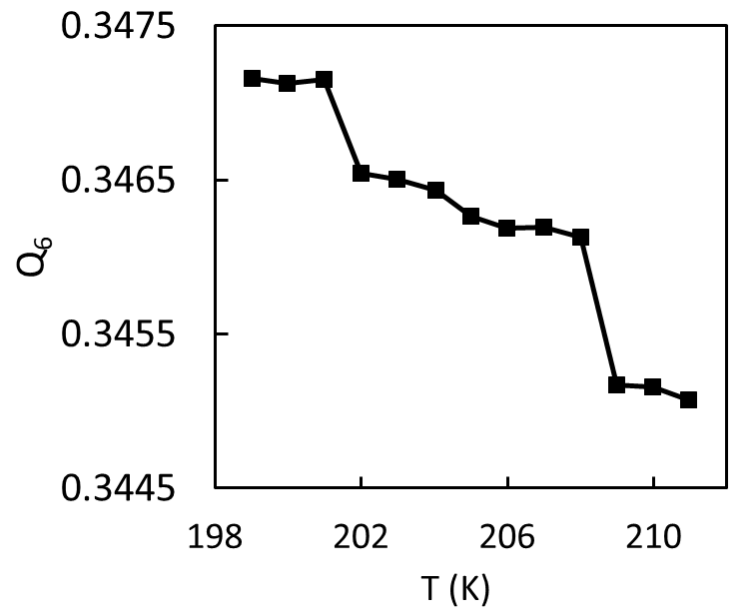

Figure S5: Bond-orientational order parameters $Q_{6}$ with $N V T$ simulations at different temperatures (a) $\rho=1.05 \mathrm{~g} / \mathrm{cm}^{3}$ (b) $\rho=0.965 \mathrm{~g} / \mathrm{cm}^{3}$.
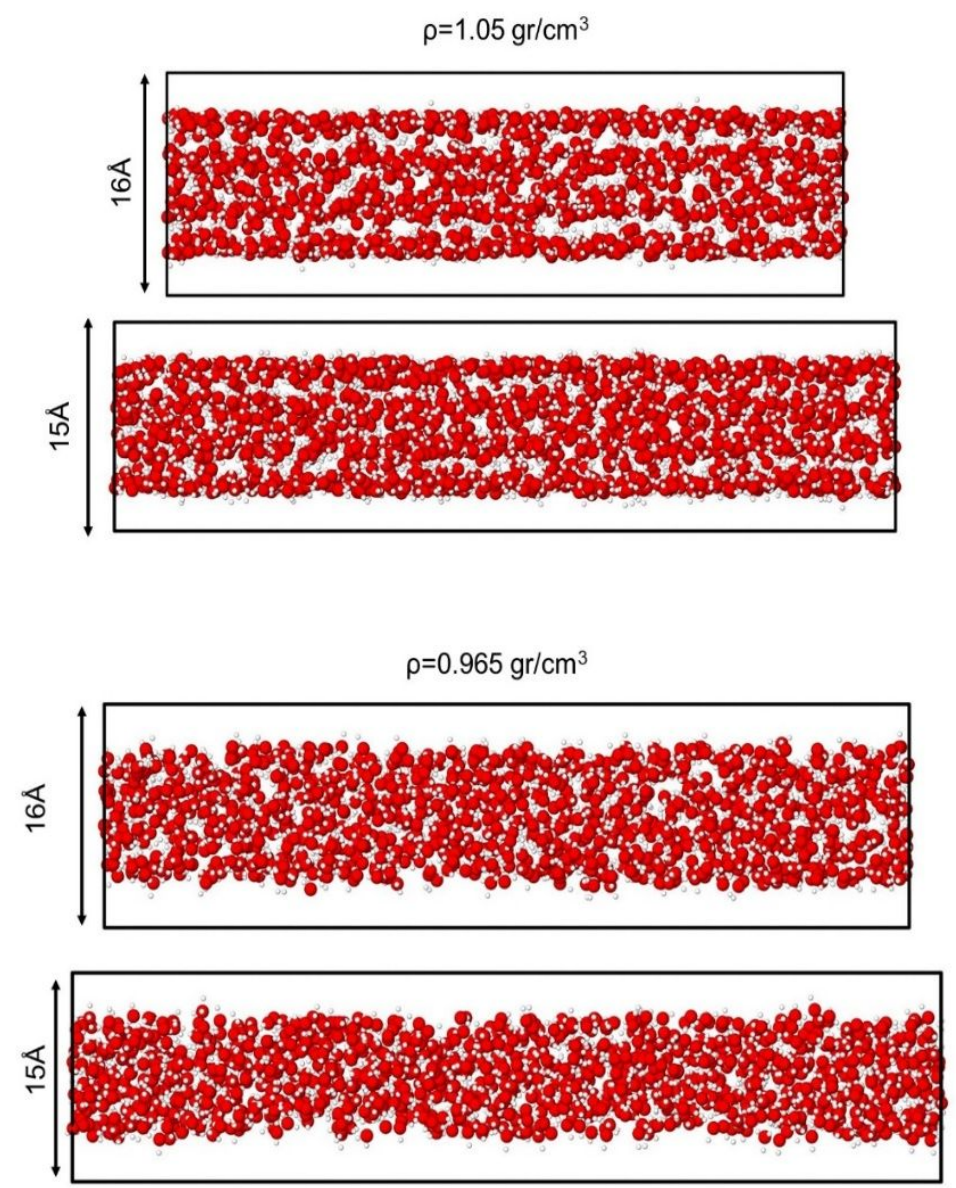

Figure S6: Side views of the confined water at two different densities with two different $\mathrm{z}$ distances. The layering structure of the confined water is maintained regardless of high and low densities. 


\section{Two systems with different densities of what we have chosen in this work:}

we decided to add two more NVT simulations results to present what is exactly happening in confined water with a bit different density than what we have reported in the manuscript. We chose two densities, first one with $\rho \sim=0.90 \mathrm{~g} / \mathrm{cm} 3$ and the second one: $\rho \sim=1.04 \mathrm{~g} / \mathrm{cm} 3$. System with $\rho$ $\sim=0.90 \mathrm{~g} / \mathrm{cm} 3$ show just one liquid-ice transition and there is no intermediate phase between these two states. System with $\rho \sim=1.04 \mathrm{~g} / \mathrm{cm} 3$ show the same behavior that we observed in the manuscript. In this case we realized that if the system is in the range of HDL/LDL density difference that we have reported for positive or negative lateral pressures, we observe this phenomenon in supercooled region $(200<\mathrm{T}<210)$ of the confined water, while if the density is far from this range, it will disappear.
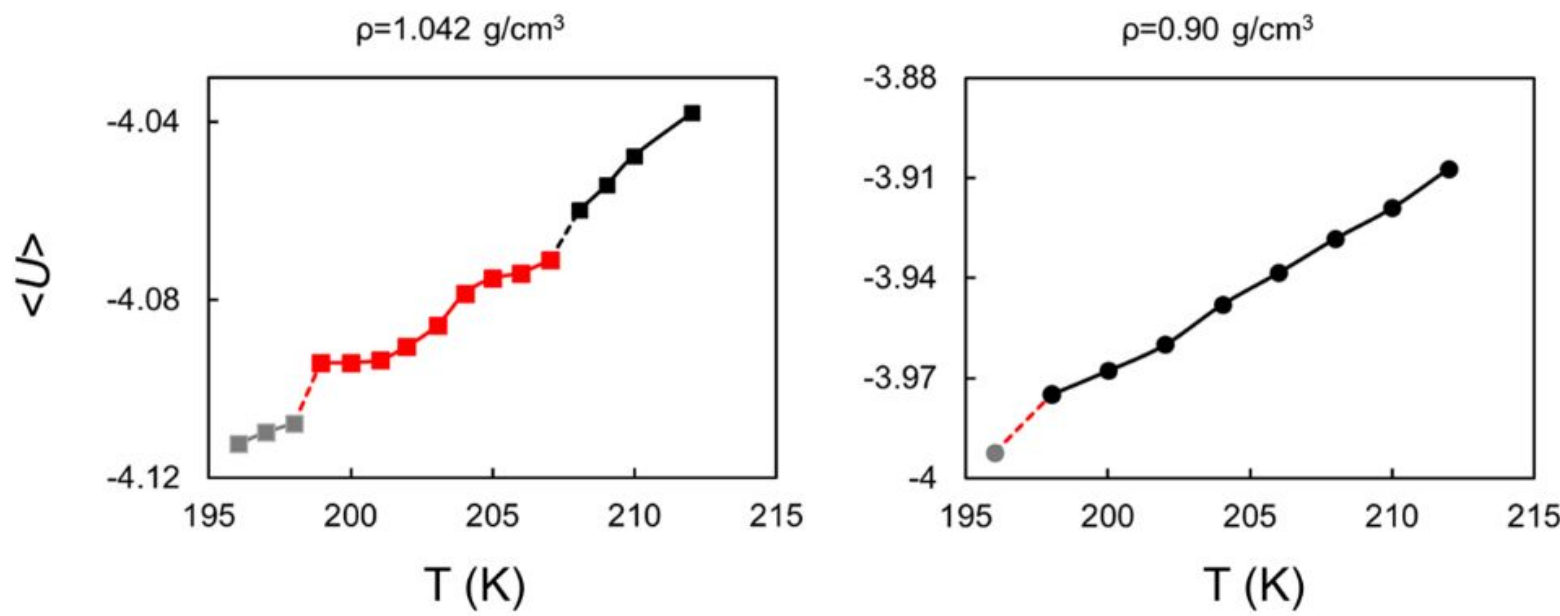

Figure S7: The potential of the confined water with NVT simulations at two different densities.

\section{CHILL+ algorithm:}

To provide evidence that no crystallization occurs in the simulations at $\mathrm{T}=202 \mathrm{~K}$ under positive and negative pressures, we have evaluated the fraction of molecules with crystalline character with one of the most common classification schemes, CHILL $+{ }^{9}$. This algorithm classifies molecules according to three crystalline structures (cubic ice, hexagonal ice, and hydrate) and two interfacial structures (interfacial ice and interfacial hydrate).

The fraction of molecules belonging to these groups at $\mathrm{T}=202 \mathrm{~K}$ along the $\rho=1.05 \mathrm{~g} / \mathrm{cm}^{3}$ and $\rho=$ $0.965 \mathrm{~g} / \mathrm{cm}^{3}$ isochore are as follows (we have collected 1000 snapshots of NVT simulations within $10 \mathrm{~ns})$ :

$\rho=1.05 \mathrm{~g} / \mathrm{cm}^{3}$ :

Hexagonal ice: $\quad 0.0 \%$

Cubic ice: $\quad 0.0 \%$

Interfacial ice: $\quad 0.4 \%$

Hydrate: $\quad 0.9 \%$

Interfacial hydrate: $\quad 2.3 \%$ 
$\rho=0.965 \mathrm{~g} / \mathrm{cm}^{3}:$

Hexagonal ice: $\quad 0.1 \%$

Cubic ice: $\quad 0.0 \%$

Interfacial ice: $\quad 1.4 \%$

Hydrate: $\quad 1.5 \%$

Interfacial hydrate: $\quad 6.8 \%$

According to the above results which have no significant ice structures, the structure is nearly isotropic.

\section{$\rho \cong 1.05 \mathrm{~g} / \mathrm{cm}^{3}$}
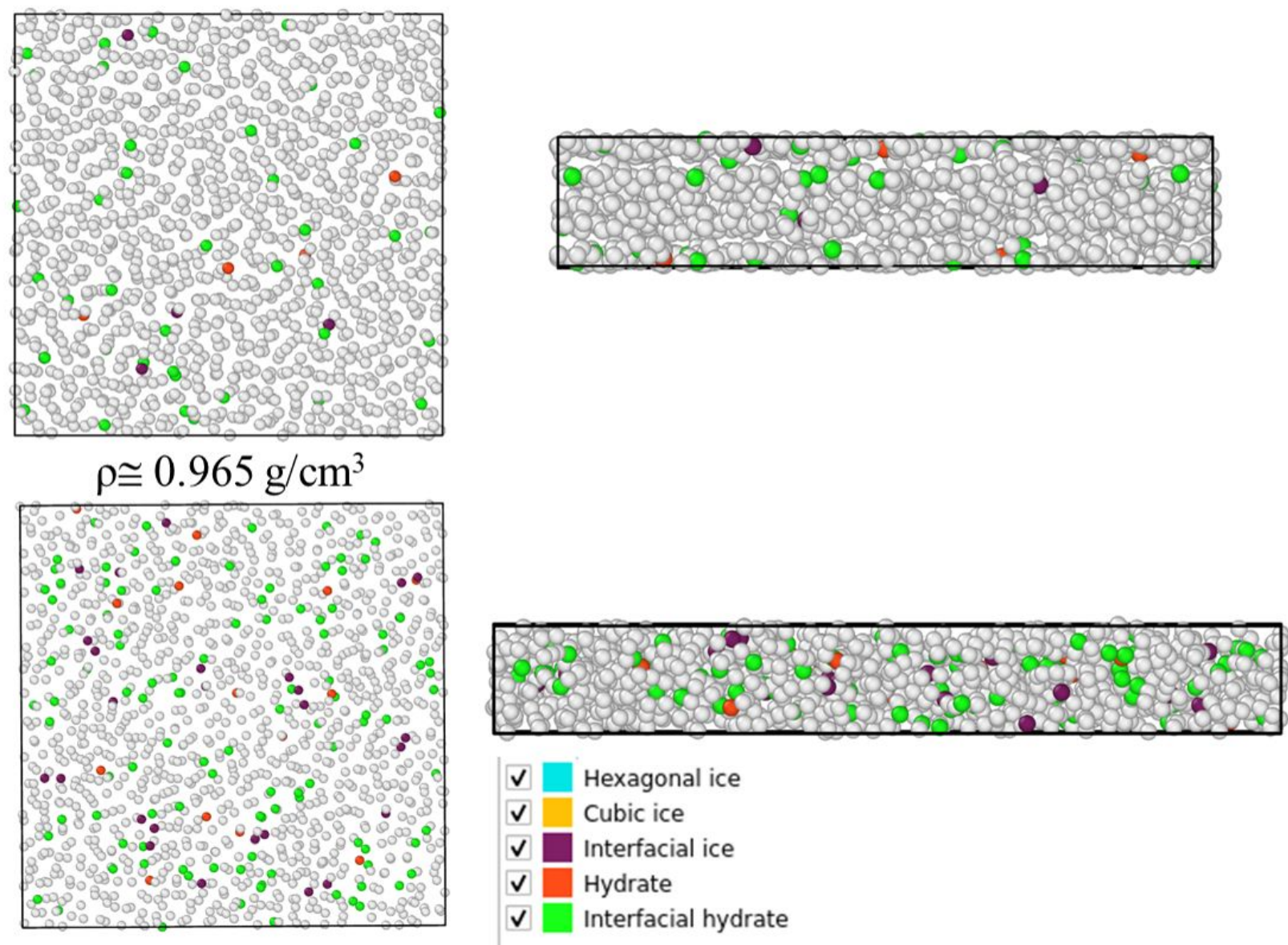

Figure S8: The top/side view snapshots by CHILL+ algorithm for two different densities. 


\section{$\underline{\text { V. References }}$}

1. Abascal, J. L. F.; Vega, C. A., General Purpose Model for the Condensed Phases of Water: TIP4P/2005. Chem. Phys. 2005, 123, 234505.

2. Jorgensen, W. L.; Chandrasekhar, J.; Madura, J. D.; Impey, R. W.; Klein, M. L., Comparison of Simple Potential Functions for Simulating Liquid Water. J. Chem. Phys. 1983, 79, 926-935.

3. Vega, C.; Abascal, J. L. F., Simulating Water with Rigid Non-Polarizable Models: a General Perspective. Phys. Chem. Chem. Phys. 2011, 13, 19663-19688.

4. Plimpton, S., Fast Parallel Algorithms for Short-Range Molecular Dynamics. Comput. Phys. 1995, 117, 1-19.

5. Hockney, R.W.; Eastwood, J.W., Computer Simulations Using Particles (McGraw-Hill, New York), pp 267-304 (1981).

6. Zwanzig, R. W., High-Temperature Equation of State by a Perturbation Method. I. Nonpolar Gases, J. Chem. Phys. 1954, 22, 1420-1426.

7. Jorgensen, W. L.; Thomas, L. L., Perspective on Free-Energy Perturbation Calculations for Chemical Equilibria, J. Chem. Theory. Comput. 2008, 4, 869-876

8. Ben-Naim, A.; Marcus, Y., Solvation Thermodynamics of Nonionic Solutes, J. Chem. Phys. 1984, 81, 2016-2017

9. Nguyen, A. H.; Morinello, V., Identification of Clathrate Hydrates, Hexagonal Ice, Cubic Ice, and Liquid Water in Simulations: the CHILL+ Algorithm, J. Phys. Chem. B 2015, 119, 93699376. 